Bull. Fac. Agric., Cairo Univ., 67: 257-264 (2016).

\title{
PERFORMANCE OF FOUR PARENTS OF MELON (Cucumis melo) AND THEIR SIX HYBRIDS
}

(Received: 15.6.2016)

\author{
By \\ M. M. Abd-Elwanis and S. Mahmoud \\ Horticulture Research Institute, Agriculture Research Center, Giza, Egypt
}

\begin{abstract}
The current study was carried out in 2014 and 2015 seasons in a plastic house at Kaha Research Farm, Kalubia Governorate. Heterosis in 6 hybrids of melon, Cucumis melo, involving 4 melon varieties, Shammam El-Jordan, Charentais, Kahera 6 and Shahd El-dokki were evaluated for average fruit weight, fruit length, fruit diameter, total soluble solids, total yield per plant, plant height, chlorophyll content, fruit shape index and fruit flesh thickness. The results of heterosis showed that the best crosses were Shammam El-Jordan $\times$ Charentais and Kahera $6 \times$ Charentais which had desirable characters. Kahera 6 variety had high GCA effect for the character plant height. Shahd Eldokki exhibited GCA effects for 2 characters, namely, average fruit weight and fruit length. Charentais variety showed high values of GCA for 2 characters, namely, total soluble solids and chlorophyll content. Estimates of SCA effects showed that the best combination was Shammam El-Jordan $\times$ Charentais for total yield per plant, flesh thickness and total soluble solids. Kahera $6 \times$ Charentais showed best SCA for plant height and average fruit weight. Heritability values for all studied characters were low in both broad and narrow sense in both 2014 and 2015 seasons.
\end{abstract}

Key words: Melon, Cucumis melo, general combining ability, specific combining ability, heritability, heterosis, yield, average fruit weight, fruit shape index, TSS.

\section{INTRODUCTION}

Melon, Cucumis melo L., is one of the most economically important vegetable crops of Cucurbitaceae. According to Luan et al. (2010), melon is a polymorphic species which is true for fruit related traits. Cultivation of $\mathrm{F}_{1}$ hybrids has a major role in the improvement of crop production and fruit quality over the past few years (Duvick, 1999). Heterosis refers to the phenomenon that $F_{1}$ hybrids exhibit phenotypic characters exceed the mean of parents. Heterosis also has an important role in the fitness of natural populations. Many researches had been carried out on heterosis and combining ability in musk melon. Exploiting of heterosis and selecting parents depending on combining ability made it more beneficial in vegetable cultivar improvement. The general combining ability (GCA) allows the identification of parents with desirable characters. Meanwhile, the SCA effects indicate the most promising hybrid combinations (Valérioet et al., 2009). Breeding for high yield and good horticultural characters of melon necessitates the evaluation and identification of varietal differences. Determination of heterosis and combining ability effects are important in identifying the best parents and combinations that could be used in a selection program to produce a new inbred line or cultivars that may possess higher quantity and quality characters such as fruit shape index, fruit firmness, total soluble solids, vitamin $\mathrm{C}$ content, flesh color and average fruit weight. Heterosis was detected for most plant and fruit characters of melon by many investigators. Hatem (1992) and Hatem et al. (1995) studying melon found heterosis for total yield as fruit weight and average fruit weight. Greish et al. (2005) studying melon reported heterosis for plant high, fruit weight, fruit length, fruit width and total soluble solids (TSS). Also, Feyzian et al. (2009a) reported heterosis for average melon fruit weight and total yield. Fernandez-Silva et al. (2009) recorded heterosis for melon fruit shape index. The main target of the current study was to compare the performance of 
the $F_{1}$ hybrids with their parents, and to estimate heterosis and combining ability to select a new local hybrid which possesses high total soluble solids, flesh firmness, small fruit core and orange flesh color.

\section{MATERIALS AND METHODS}

The current study on Melon, Cucumis melo, was conducted at Kaha Vegetable Research Farm, Kalubia Governorate during the seasons of 2014 and 2015 in a plastic house. Four genetically diverse varieties of melon, viz., Shammam ElJordan, Shahd Eldokki, Kahera 6 and Charentais were provided by the Vegetable Research Department, Horticulture Research Institute, Agriculture Research Center, Ministry of Agriculture Egypt. The four melon varieties were crossed in one direction to produce $6 \mathrm{~F}_{1}$ hybrids in a plastic house in September of 2013. Descriptions of these varieties are shown in Table (1). All the four parents and the $6 F_{1}$ hybrids were grown for evaluation in two seasons in September of 2014 and 2015 in a randomized complete block design with three replicates. Each plot contained 20 plants, 10 on each ridge with $50 \mathrm{~cm}$ between estimated over the mid-parent and high-parent as percentage. Combining ability and heritability in broad and narrow sense were estimated according to Griffing (1956) as described by Singh and Chaudhary (1977).

$$
\begin{aligned}
\mathrm{H} \% \%_{\mathrm{MP}}= & \frac{\overline{\mathrm{F}}_{1}-\overline{\mathrm{MP}}}{\overline{\mathrm{MP}}} \times 100 \\
& \mathrm{H} \%_{\mathrm{HP}}=\frac{\overline{\mathrm{F}_{1}}-\overline{\mathrm{HP}}}{\overline{\mathrm{HP}}} \times 100
\end{aligned}
$$

Where:

$\mathrm{H} \%_{\mathrm{MP}}=$ heterosis over mid - parent

$\overline{\mathrm{F}}_{1}=$ hybrid mean

$\overline{\mathrm{MP}}=$ two parents mean

$\mathrm{H} \%_{\mathrm{HP}}=$ heterosis over high - parent

$\overline{\mathrm{HP}}=$ high parent mean

$$
\begin{aligned}
& \mathrm{H}^{2}=\frac{\mathrm{V}_{\mathrm{A}}+\mathrm{V}_{\mathrm{D}}}{\mathrm{V}_{\mathrm{G}}+\mathrm{V}_{\mathrm{E}}} \\
& \mathrm{h}^{2}=\frac{\mathrm{V}_{\mathrm{A}}}{\mathrm{V}_{\mathrm{G}}+\mathrm{V}_{\mathrm{E}}}
\end{aligned}
$$

\begin{tabular}{|c|c|c|c|c|c|}
\hline \multicolumn{2}{|c|}{ Genotype name } & \multirow{2}{*}{$\begin{array}{l}\text { Country } \\
\text { of origin }\end{array}$} & \multirow{2}{*}{ Source } & \multirow{2}{*}{ Fruit shuck color } & \multirow{2}{*}{$\begin{array}{l}\text { Fruit flesh } \\
\text { color }\end{array}$} \\
\hline Common & Scientific & & & & \\
\hline $\begin{array}{l}\text { Shammam El- } \\
\text { Jordan }\end{array}$ & Cucumis melo & Jordan & $\begin{array}{c}\text { Horticulture } \\
\text { research institute }\end{array}$ & Light green, with net & $\begin{array}{c}\text { Reddish } \\
\text { orange }\end{array}$ \\
\hline Shahd ElDokki & C. melo & Egypt & $\begin{array}{c}\text { Horticulture } \\
\text { research institute }\end{array}$ & $\begin{array}{c}\text { Sandy yellow, with } \\
\text { narrow net }\end{array}$ & $\begin{array}{c}\text { Greenish } \\
\text { white }\end{array}$ \\
\hline Kahera 6 & $\begin{array}{l}\text { C. melo var } \\
\text { aegyptiacus }\end{array}$ & Egypt & $\begin{array}{l}\text { Horticulture } \\
\text { research institute }\end{array}$ & $\begin{array}{c}\text { Dark beige with } \\
\text { bluish green strips, } \\
\text { without net }\end{array}$ & $\begin{array}{l}\text { Greenish } \\
\text { white }\end{array}$ \\
\hline Charentais & C. melo & France & $\begin{array}{c}\text { Horticulture } \\
\text { research institute }\end{array}$ & $\begin{array}{l}\text { Dark beige with green } \\
\text { strips, with narrow net }\end{array}$ & Dark orange \\
\hline
\end{tabular}

Table (1): Description of parental varieties of melon used in the current study.

plants. All agricultural practices were followed according to the recommendations of the Ministry of Agriculture and Land Reclamation, Egypt. Data were recorded on 10 randomly selected plants and 10 fruits per plant in each plot for total yield per plant, plant height, chlorophyll content in plant leaves, fruit length, fruit diameter, fruit shape index, fruit flesh thickness, total soluble solids and average fruit weight. Data were statistically analyzed according to Snedecor and Cochran (1980). Heterosis expressed by the $F_{1}$ hybrids was
Where:

$\mathrm{H}^{2}=$ heritability in broad sense

$\mathrm{h}^{2}=$ heritability in narrow sense

$\mathrm{V}_{\mathrm{A}}=$ Additive variance

$\mathrm{V}_{\mathrm{D}}=$ Dominance variance

$\mathrm{V}_{\mathrm{G}}=$ Genetic variance

$\mathrm{V}_{\mathrm{E}}=$ Environmental variance

\section{RESULTS AND DISCUSSION}

Regarding the data presented in Tables ( 2 and 3 ), there were significant differences among 
genotypes for all the studied characters in both seasons. Wide range of variation was found among the selected parents. Parents in each cross differed for growth habit, fruit size and shape. In respect to the mean performance, the highest parents were Shahd ElDokki for total yield per plant, average fruit weight and fruit length and Kahera 6 for plant height and total soluble solids. The highest $F_{1}$ hybrids were Shammam El-Jordan $\times$ Charentais for total yield per plant, Shahd ElDokki $\times$ Kahera 6 for average fruit weight and Kahera $6 \times$ Charentais for plant height.

Data in Table (3) showed the mean squares for analysis of variance of parents, $F_{1}$ hybrids, general and specific combining ability where there were significant differences for all the studied characters. These results agreed with those of Kupper and Jack (1988) who found highly significant differences of GCA and SCA for yield and its components in cucumber. GCA/SCA ratio was more than one for plant height, total yield per plant, average fruit weight and fruit length indicating that additive gene effects were more important in the inheritance of these characters. While, the ratio was less than one for fruit diameter indicating that non-additive gene effects were important in the inheritance of this character. This was in accordance with the results obtained by Feyzian et al. (2009b) in melon. Variance due to GCA was higher than that due to SCA for plant height, total yield per plant, average fruit weight and fruit length. This indicates the the additive type of gene action is more important than nonadditive type in the inheritance of these characters and supporting the results obtained on GCA/SCA ratio.

Estimates of heterosis over mid-parent and high-parent are presented in Tables (4a and 4b) show significant differences in both seasons. In respect to heterosis over mid-parent, the highest heterosis values were estimated for the hybrids Shammam El-Jordan $\times$ Shahd El-Dokki for plant height, Shammam El-Jordan $\times$ Charentais for total yield per plant, flesh thickness and total soluble solids and Kahera $6 \times$ Charentais for average fruit weight. The values of heterosis over mid-parents showed that out of the six evaluated hybrids the crosses that surpassed their mid-parents were 5 hybrids (Shammam El-Jordan $\times$ Shahd El-Dokki, Shammam El-Jordan $\times$ Kahera 6, Shammam El-
Jordan $\times$ Charentais, Shahd El-Dokki $\times$ Kahera 6 and Kahera $6 \times$ Charentais) for plant height, 2 hybrids (Shammam El-Jordan $\times$ Charentais and Kahera $6 \times$ Charentais) for total yield per plant, 1 hybrid (Kahera $6 \times$ Charentais) for average fruit weight, 5 hybrids (Shammam El-Jordan $\times$ Kahera 6, Shammam El-Jordan $\times$ Charentais, Shahd ElDokki $\times$ Kahera 6, Shahd ElDokki $\times$ Charentais and Kahera $6 \times$ Charentais) for fruit length, 3 hybrids (Shammam El-Jordan $\times$ Kahera 6, Shammam El-Jordan $\times$ Charentais and Kahera $6 \times$ Charentais) for fruit diameter, 3 hybrids $($ Shammam El-Jordan $\times$ Shahd El-Dokki, Shahd ElDokki $\times$ Kahera 6 and Shahd El-Dokki $\times$ Charentais) for fruit shape index, 2 hybrids (Shammam El-Jordan $\times$ Kahera 6 and Shammam El-Jordan $\times$ Charentais) for flesh thickness, 1 hybrid (Shammam El-Jordan $\times$ Charentais) for total soluble solids and 3 hybrids (Shammam ElJordan $\times$ Kahera 6, Shahd El-Dokki $\times$ Kahera 6 and Shahd El-Dokki $\times$ Charentais) for chlorophyll content. The range of increase in the hybrids over their mid-parents (Table 4) was between $18.49 \%$ (Shammam El-Jordan $\times$ Kahera6 for Chlorophyll content) to $125.24 \%$ (Shammam El-Jordan $\times$ Charantais) for total yield per plant in the first season while it was $1.09 \%$ (Shammam El-Jordan $\times$ Charentais for total soluble solids) in the second one. Only the hybrid Kahera $6 \times$ Charentais showed an increase over the mid-parents in the total yield per plant and average fruit weight. In respect to heterosis over high-parent, the highest heterosis values were estimated for the hybrids Shammam El-Jordan $\times$ Shahd El-Dokki average fruit weight (2015 season), fruit length (2014 season) and fruit shape index (2014 and 2015 seasons); Shammam El-Jordan $\times$ Kahera 6 for fruit diameter (2014 and 2015 seasons), flesh thickness (2015 season) and chlorophyll content (2015 season); Shammam El-Jordan $\times$ Charantais for total yield per plant (2014 and 2015 seasons), flesh thickness (2014 season) and total soluble solids (2014 season); Shahd ElDokki $\times$ Kahera 6 for chlorophyll content (2015 season) and Kahera 6 $\times$ Charentais for plant height (2014 and 2015 seasons), average fruit weight (2014 season), fruit length (2015 season) and total soluble solids (2015 season). The crosses that surpassed their highparents were Shammam El-Jordan $\times$ Shahd ElDokki for fruit shape index, Shammam El-Jordan 
Table (2): Mean performance of the 4 parents and their $6 F_{1}$ hybrids in 2014 and 2015 seasons.

\begin{tabular}{|c|c|c|c|c|c|c|c|c|c|c|c|c|c|c|c|c|c|c|}
\hline \multirow[t]{2}{*}{ Genotypes } & \multicolumn{2}{|c|}{$\begin{array}{l}\text { Plant height } \\
\quad(\mathrm{cm})\end{array}$} & \multicolumn{2}{|c|}{$\begin{array}{l}\text { Total yield per } \\
\text { plant (kg) }\end{array}$} & \multicolumn{2}{|c|}{$\begin{array}{c}\text { Average fruit } \\
\text { weight (kg) }\end{array}$} & \multicolumn{2}{|c|}{$\begin{array}{l}\text { Fruit length } \\
\quad(\mathrm{cm})\end{array}$} & \multicolumn{2}{|c|}{$\begin{array}{l}\text { Fruit diameter } \\
\qquad(\mathbf{c m})\end{array}$} & \multicolumn{2}{|c|}{$\begin{array}{l}\text { Fruit shape } \\
\text { index }\end{array}$} & \multicolumn{2}{|c|}{$\begin{array}{l}\text { Flesh thickness } \\
(\mathrm{cm})\end{array}$} & \multicolumn{2}{|c|}{$\begin{array}{c}\text { Total soluble } \\
\text { solids }(\%)\end{array}$} & \multicolumn{2}{|c|}{$\begin{array}{c}\text { Chlorophyll } \\
\text { content in } \\
\text { leaves }(\%)\end{array}$} \\
\hline & 2014 & 2015 & 2014 & 2015 & 2014 & 2015 & 2014 & 2015 & 2014 & 2015 & 2014 & 2015 & 2014 & 2015 & 2014 & 2015 & 2014 & 2015 \\
\hline 1 & 300 & 283.67 & 2.27 & 3.83 & 1.47 & 1.38 & 13 & 13.17 & 10 & 10.5 & 1.33 & 1.26 & 2.17 & 2.33 & 7.67 & 8 & 36.63 & 43.43 \\
\hline 2 & 354.67 & 363.33 & 14.23 & 10.28 & 2.39 & 2.27 & 16.67 & 18.67 & 10.67 & 18 & 1.58 & 1.04 & 3.33 & 4 & 6.5 & 7.67 & 39.4 & 41.47 \\
\hline 3 & 374 & 383 & 2.95 & 5.37 & 1.08 & 1 & 12.33 & 12.33 & 8.4 & 8.4 & 1.47 & 1.47 & 2.93 & 2.93 & 10.67 & 10.67 & 37.63 & 39.43 \\
\hline 4 & 361 & 366.33 & 8.67 & 9.95 & 0.76 & 0.68 & 13.5 & 14.67 & 11.37 & 12.17 & 1.19 & 1.21 & 3.67 & 3.47 & 8.33 & 10.33 & 47.5 & 47.53 \\
\hline $1 \times 2$ & 404 & 414.33 & 7.47 & 6.43 & 1.11 & 1.03 & 20 & 14.1 & 10.17 & 8.83 & 1.97 & 1.6 & 3.67 & 2.53 & 13 & 6.2 & 41.33 & 39.63 \\
\hline $1 \times 3$ & 377 & 391.33 & 2.4 & 3.93 & 0.76 & 0.68 & 13.83 & 15.03 & 11.5 & 12.13 & 1.21 & 1.24 & 3 & 2.87 & 13 & 6.53 & 44 & 46.4 \\
\hline $1 \times 4$ & 351 & 375.33 & 12.32 & 13.31 & 0.59 & 0.51 & 13.33 & 17.77 & 11.77 & 11.7 & 1.13 & 1.52 & 4.5 & 3.3 & 15 & 9.27 & 35.8 & 42.9 \\
\hline $2 \times 3$ & 431.33 & 428 & 3.92 & 2.43 & 1.33 & 1.25 & 17.33 & 16.33 & 10.17 & 10.5 & 1.71 & 1.55 & 3.6 & 3.1 & 10 & 7 & 43.87 & 45.63 \\
\hline $2 \times 4$ & 377 & 384.67 & 9.55 & 6.35 & 0.75 & 0.67 & 16.17 & 17.2 & 10 & 10.57 & 1.63 & 1.64 & 300 & 3.53 & 12.67 & 8.87 & 49.67 & 44.87 \\
\hline $3 \times 4$ & 447.33 & 446 & 6.5 & 9.95 & 1.1 & 1.02 & 13.83 & 18 & 12.77 & 11.03 & 1.08 & 1.64 & 2.83 & 3 & 14 & 10 & 46.77 & 41.97 \\
\hline $\begin{array}{c}\text { LSD at } \\
5 \%\end{array}$ & 13.51 & 28.59 & 3.62 & 3.4 & 0.05 & 0.05 & 4.03 & 1.57 & 1.75 & 1.15 & 0.45 & 0.22 & 1.03 & 0.4 & 3.47 & 1.14 & 8.42 & 5.48 \\
\hline
\end{tabular}

1: Shammam El-Jordan, 2: ShahdEl-Dokki, 3: Kahera 6 and 4: Charentais.

Table (3): Mean square for analysis of variance of genotypes (parents and $\mathrm{F}_{1}$ ) and combining ability (GCA and SCA) of melon in 2014 and 2015 seasons.

\begin{tabular}{|c|c|c|c|c|c|c|c|c|c|c|c|c|c|c|c|c|c|c|c|}
\hline \multirow{2}{*}{$\begin{array}{l}\text { Source of } \\
\text { Variance }\end{array}$} & \multirow[t]{2}{*}{ df } & \multicolumn{2}{|c|}{ Plant height } & \multicolumn{2}{|c|}{$\begin{array}{c}\text { Total yield per } \\
\text { plant }\end{array}$} & \multicolumn{2}{|c|}{$\begin{array}{l}\text { Average fruit } \\
\text { weight }\end{array}$} & \multicolumn{2}{|c|}{ Fruit length } & \multicolumn{2}{|c|}{$\begin{array}{c}\text { Fruit } \\
\text { diameter }\end{array}$} & \multicolumn{2}{|c|}{$\begin{array}{c}\text { Fruit shape } \\
\text { index }\end{array}$} & \multicolumn{2}{|c|}{ Flesh thickness } & \multicolumn{2}{|c|}{$\begin{array}{l}\text { Total soluble } \\
\text { solids }\end{array}$} & \multicolumn{2}{|c|}{$\begin{array}{c}\text { Chlorophyll } \\
\text { content in leaves }\end{array}$} \\
\hline & & 2014 & 2015 & 2014 & 2015 & 2014 & 2015 & 2014 & 2015 & 2014 & 2015 & 2014 & 2015 & 2014 & 2015 & 2014 & 2015 & 2014 & 2015 \\
\hline \multicolumn{20}{|c|}{ Mean squares for analysis of variance of genotypes } \\
\hline Genotypes & 9 & $5330.43 *$ & $5878.28 *$ & $53.27 *$ & $37.14^{*}$ & $3.19 *$ & $2.8^{*}$ & $17.81^{*}$ & $14.28^{*}$ & $4.44^{*}$ & $20.94 *$ & $0.25^{*}$ & $0.14 *$ & $1.2 *$ & $0.72 *$ & $25.1 *$ & $7.79 *$ & $70.69 *$ & $22.56 \mathrm{~ns}$ \\
\hline & 18 & 62.04 & 277.74 & 4.44 & 3.92 & 0.001 & 0.001 & 5.53 & 1.04 & 0.74 & 0.45 & 0.07 & 0.02 & 0.36 & 0.06 & 4.09 & 0.44 & 24.09 & 10.21 \\
\hline \multicolumn{20}{|c|}{ Mean squares for analysis of variance of combining ability (General, GCA and Specific, SCA) } \\
\hline GCA & 3 & $2162.98 *$ & $2407.25^{*}$ & $36.43^{*}$ & $15.81^{*}$ & $0.46^{*}$ & $0.45^{*}$ & $10.76^{*}$ & $5.77^{*}$ & $1.26^{*}$ & $7.41 *$ & $0.15^{*}$ & $0.01 \mathrm{~ns}$ & $0.22 \mathrm{~ns}$ & $0.52 *$ & $3.52 \mathrm{~ns}$ & $5.07 *$ & $30.39 *$ & $5.14 \mathrm{~ns}$ \\
\hline SCA & 6 & $1583.73 *$ & $1735.52 *$ & $8.42 *$ & $10.67 *$ & $0.18^{*}$ & $0.17^{*}$ & $3.53^{*}$ & $4.25^{*}$ & $1.59 *$ & $6.76^{*}$ & $0.05 \mathrm{~ns}$ & $0.06^{*}$ & $0.49 *$ & $0.10^{*}$ & $10.79 *$ & $1.36^{*}$ & $20.15 n s$ & $8.71 \mathrm{~ns}$ \\
\hline Error & 18 & 20.68 & 92.58 & 1.48 & 1.31 & 0.0003 & 0.0003 & 1.84 & 0.35 & 0.25 & 0.15 & 0.02 & 0.01 & 0.12 & 0.02 & 1.36 & 0.15 & 8.03 & 3.4 \\
\hline \multicolumn{2}{|c|}{ GCA/SCA } & 1.37 & 1.39 & 4.33 & 1.48 & 2.63 & 2.63 & 3.05 & 1.36 & 0.79 & 1.1 & 2.79 & 0.17 & 0.44 & 5.12 & 0.33 & 3.74 & 1.51 & 0.99 \\
\hline \multirow{2}{*}{\multicolumn{2}{|c|}{$\begin{array}{l}\dot{\mathrm{O}}_{\mathrm{g}}^{2} \\
\mathrm{O}^{2^{2}}\end{array}$}} & 96.54 & 111.95 & 4.67 & 0.86 & 0.05 & 0.05 & 1.2 & 0.25 & -0.06 & 0.11 & 0.02 & -0.01 & -0.05 & 0.07 & -1.21 & 0.62 & 1.71 & -0.95 \\
\hline & & 1563.05 & 1642.94 & 6.94 & 9.36 & 0.18 & 0.17 & 1.69 & 3.9 & 1.35 & 6.61 & 0.03 & 0.06 & 0.37 & 0.08 & 9.42 & 1.21 & 12.12 & 5.31 \\
\hline
\end{tabular}

* significant at $5 \%$ 
Performance of four parents of melon

Table (4a): Heterosis over mid-parent (\%) of $F_{1}$ hybrids in 2014 and 2015 seasons.

\begin{tabular}{|c|c|c|c|c|c|c|c|c|c|c|c|c|c|c|c|c|c|c|}
\hline \multirow[t]{2}{*}{ Genotype } & \multicolumn{2}{|c|}{ Plant height } & \multicolumn{2}{|c|}{ Total yield per plant } & \multicolumn{2}{|c|}{ Average fruit weight } & \multicolumn{2}{|c|}{ Fruit length } & \multicolumn{2}{|c|}{ Fruit diameter } & \multicolumn{2}{|c|}{ Fruit shape index } & \multicolumn{2}{|c|}{ Flesh thickness } & \multicolumn{2}{|c|}{$\begin{array}{c}\begin{array}{c}\text { Total soluble } \\
\text { solids }\end{array} \\
\end{array}$} & \multicolumn{2}{|c|}{$\begin{array}{c}\begin{array}{c}\text { Chlorophyll content in } \\
\text { leaves }\end{array} \\
\end{array}$} \\
\hline & 2014 & 2015 & 2014 & 2015 & 2014 & 2015 & 2014 & 2015 & 2014 & 2015 & 2014 & 2015 & 2014 & 2015 & 2014 & 2015 & 2014 & 2015 \\
\hline $1 \times 2$ & $\begin{array}{c}23.42 \\
a\end{array}$ & $\begin{array}{c}28.08 \\
n s\end{array}$ & $\begin{array}{c}-9.49 \\
\text { abd }\end{array}$ & $\begin{array}{l}-8.95 \\
\mathrm{abc}\end{array}$ & $\begin{array}{l}-42.56 \\
\text { a-d }\end{array}$ & $\begin{array}{c}-48.82 \\
\mathrm{a}\end{array}$ & $\begin{array}{c}34.83 \\
\mathrm{a}\end{array}$ & $\begin{array}{c}-11.41 \\
\text { a-e }\end{array}$ & $\begin{array}{l}-1.61 \\
\mathrm{a}-\mathrm{e}\end{array}$ & $\begin{array}{c}-38.01 \\
\text { a-e }\end{array}$ & $\begin{array}{c}35.85 \\
\mathrm{a}\end{array}$ & $\begin{array}{c}39.03 \\
\mathrm{ab}\end{array}$ & $\begin{array}{c}33.33 \\
\mathrm{ab}\end{array}$ & $\begin{array}{l}-20 \\
\mathrm{a}-\mathrm{e}\end{array}$ & $\begin{array}{c}83.53 \\
\mathrm{ab}\end{array}$ & $\begin{array}{c}-20.85 \\
\text { a-d }\end{array}$ & $\begin{array}{c}8.72 \\
\mathrm{ae}\end{array}$ & $\begin{array}{l}-6.16 \\
a b c\end{array}$ \\
\hline $1 \times 3$ & $\begin{array}{c}\mathrm{a} \\
11.87 \\
\mathrm{~ns}\end{array}$ & $\begin{array}{c}17.4 \\
\text { ns }\end{array}$ & $\begin{array}{c}-7.99 \\
a b c\end{array}$ & $\begin{array}{c}-14.64 \\
\text { a-d }\end{array}$ & $\begin{array}{c}-40.31 \\
a b c\end{array}$ & $\begin{array}{c}-42.85 \\
a b\end{array}$ & $\begin{array}{c}9.21 \\
\mathrm{ab}\end{array}$ & $\begin{array}{c}17.91 \\
\text { abc }\end{array}$ & $25 \mathrm{ab}$ & $\begin{array}{c}\mathrm{a}-\mathrm{c} \\
28.4 \\
\mathrm{a}\end{array}$ & $\begin{array}{c}-13.48 \\
a-e\end{array}$ & $\begin{array}{l}-9.18 \\
\text { a-e }\end{array}$ & $\begin{array}{c}17.65 \\
\text { abc }\end{array}$ & $\begin{array}{c}a-86 \\
\mathrm{ab}\end{array}$ & $\begin{array}{c}41.82 \\
\text { a-e }\end{array}$ & $\begin{array}{l}\text { a-a } \\
-30 \\
a-e\end{array}$ & $\begin{array}{c}18.49 \\
\mathrm{a}\end{array}$ & $\begin{array}{c}11.99 \\
\mathrm{~b}\end{array}$ \\
\hline $1 \times 4$ & $\begin{array}{l}6.2 \\
a b c\end{array}$ & $\begin{array}{c}15.49 \\
\mathrm{~ns}\end{array}$ & $\begin{array}{c}125.24 \\
\mathrm{a}\end{array}$ & $\begin{array}{c}93.13 \\
\mathrm{a}\end{array}$ & $\begin{array}{c}-46.75 \\
\text { a-e }\end{array}$ & $\begin{array}{c}-50.22 \\
\mathrm{abc}\end{array}$ & $\begin{array}{c}0.63 \\
a b c\end{array}$ & $\begin{array}{c}27.66 \\
\text { ab }\end{array}$ & $\begin{array}{c}10.14 \\
a b c\end{array}$ & $\begin{array}{l}3.24 \\
\mathrm{abc}\end{array}$ & $\begin{array}{c}-10.1 \\
\text { a-d }\end{array}$ & $\begin{array}{c}23.19 \\
\text { a-d }\end{array}$ & $\begin{array}{c}54.29 \\
\mathrm{a}\end{array}$ & $\begin{array}{c}13.79 \\
\mathrm{a}\end{array}$ & $\begin{array}{c}87.5 \\
\mathrm{a}\end{array}$ & $1.09 \mathrm{a}$ & $\begin{array}{c}-14.9 \\
\text { a-e }\end{array}$ & $\begin{array}{c}-5.68 \\
a b c\end{array}$ \\
\hline $2 \times 3$ & $\begin{array}{c}18.39 \\
\mathrm{c}\end{array}$ & $\begin{array}{c}14.69 \\
\text { ns }\end{array}$ & $\begin{array}{c}-54.41 \\
\mathrm{a}-\mathrm{e}\end{array}$ & $\begin{array}{c}-68.9 \\
\text { a-e }\end{array}$ & $\begin{array}{c}-23.45 \\
\mathrm{ab}\end{array}$ & $\begin{array}{c}-23.85 \\
\text { a-d }\end{array}$ & $\begin{array}{c}19.54 \\
\text { abd }\end{array}$ & $\begin{array}{c}5.38 \\
\text { a-d }\end{array}$ & $\begin{array}{c}6.46 \\
\text { a-d }\end{array}$ & $\begin{array}{c}-20.45 \\
\text { a-d }\end{array}$ & $\begin{array}{c}12.02 \\
a b c\end{array}$ & $\begin{array}{c}23.74 \\
\mathrm{abc}\end{array}$ & $\begin{array}{c}14.89 \\
\text { a-d }\end{array}$ & $\begin{array}{c}10.58 \\
\text { a-e }\end{array}$ & $\begin{array}{c}16.5 \\
a-e\end{array}$ & $\begin{array}{c}-23.64 \\
\text { a-e }\end{array}$ & $\begin{array}{c}13.89 \\
\mathrm{c}\end{array}$ & $\begin{array}{c}12.81 \\
\mathrm{a}\end{array}$ \\
\hline $2 \times 4$ & $\begin{array}{l}5.36 \\
a b c\end{array}$ & $\begin{array}{c}5.44 \\
\mathrm{~ns}\end{array}$ & $\begin{array}{c}-16.61 \\
a-e\end{array}$ & $\begin{array}{c}-37.23 \\
\mathrm{a}-\mathrm{e}\end{array}$ & $\begin{array}{c}-52.4 \\
\text { a-e }\end{array}$ & $\begin{array}{c}-54.73 \\
\text { a-e }\end{array}$ & $\begin{array}{l}7.18 \\
\text { abe }\end{array}$ & $\begin{array}{l}3.2 \\
\text { a-e }\end{array}$ & $\begin{array}{l}-9.23 \\
\text { a-e }\end{array}$ & $\begin{array}{c}-29.94 \\
\text { a-e }\end{array}$ & $\begin{array}{c}17.49 \\
\mathrm{ab}\end{array}$ & $\begin{array}{l}45.65 \\
\mathrm{a}\end{array}$ & $\begin{array}{c}-14.29 \\
\text { a-d }\end{array}$ & $\begin{array}{c}-5.38 \\
a b c\end{array}$ & $\begin{array}{c}70.79 \\
\text { abc }\end{array}$ & $\begin{array}{c}-1.48 \\
\mathrm{ab}\end{array}$ & $\begin{array}{c}14.31 \\
\mathrm{~b}\end{array}$ & $\begin{array}{r}0.82 \\
a b c\end{array}$ \\
\hline $3 \times 4$ & $\begin{array}{c}21.72 \\
\mathrm{~b}\end{array}$ & $\begin{array}{c}19.04 \\
\text { ns }\end{array}$ & $\begin{array}{c}11.88 \\
\mathrm{ab}\end{array}$ & $\begin{array}{c}29.97 \\
a b\end{array}$ & $\begin{array}{c}20.19 \\
\mathrm{a} \\
\end{array}$ & $\begin{array}{c}22.11 \\
\text { a-e }\end{array}$ & $\begin{array}{c}7.1 \mathrm{a}- \\
\mathrm{e}\end{array}$ & $\begin{array}{c}33.33 \\
\mathrm{a} \\
\end{array}$ & $\begin{array}{c}29.17 \\
\mathrm{a} \\
\end{array}$ & $\begin{array}{c}7.29 \\
\mathrm{ab} \\
\end{array}$ & $\begin{array}{c}-18.54 \\
\text { a-e }\end{array}$ & $\begin{array}{c}22.5 \\
\mathrm{a}-\mathrm{e} \\
\end{array}$ & $\begin{array}{c}-14.14 \\
\text { a-d }\end{array}$ & $\begin{array}{c}-6.25 \\
\text { a-d } \\
\end{array}$ & $\begin{array}{c}47.37 \\
\text { a-d }\end{array}$ & $\begin{array}{c}-4.76 \\
a b c\end{array}$ & $\begin{array}{c}9.87 \\
\mathrm{ad} \\
\end{array}$ & $\begin{array}{c}-3.49 \\
\mathrm{ab} \\
\end{array}$ \\
\hline CD at $5 \%$ & 11.70 & 24.76 & 3.13 & 2.94 & 0.05 & 0.04 & 3.49 & 1.52 & 1.28 & 1.00 & 0.39 & 0.19 & 0.90 & 0.35 & 3.01 & 0.98 & 7.29 & 4.75 \\
\hline
\end{tabular}

1: Shammam El-Jordan, 2: ShahdEl-Dokki, 3: Kahera 6 and 4: Charentais.

Table (4b): Heterosis over high-parent (\%) of $F_{1}$ hybrids in 2014 and 2015 seasons.

\begin{tabular}{|c|c|c|c|c|c|c|c|c|c|c|c|c|c|c|c|c|c|c|}
\hline \multirow[t]{2}{*}{ Genotype } & \multicolumn{2}{|c|}{ Plant height } & \multicolumn{2}{|c|}{ Total yield per plant } & \multicolumn{2}{|c|}{$\begin{array}{c}\text { Average fruit } \\
\text { weight }\end{array}$} & \multicolumn{2}{|c|}{ Fruit length } & \multicolumn{2}{|c|}{ Fruit diameter } & \multicolumn{2}{|c|}{ Fruit shape index } & \multicolumn{2}{|c|}{ Flesh thickness } & \multicolumn{2}{|c|}{ Total soluble solids } & \multicolumn{2}{|c|}{$\begin{array}{c}\text { Chlorophyll content in } \\
\text { leaves }\end{array}$} \\
\hline & 2014 & 2015 & 2014 & 2015 & 2014 & 2015 & 2014 & 2015 & 2014 & 2015 & 2014 & 2015 & 2014 & 2015 & 2014 & 2015 & 2014 & 2015 \\
\hline $1 \times 2$ & $\begin{array}{c}13.91 \\
\mathrm{c}\end{array}$ & $\begin{array}{c}14.04 \\
\text { ns }\end{array}$ & $\begin{array}{c}-47.54 \\
\text { a-e }\end{array}$ & $\begin{array}{c}-37.5 \\
a-d\end{array}$ & $\begin{array}{c}-53.61 \\
a-d\end{array}$ & $\begin{array}{c}2.16 \\
\mathrm{a}\end{array}$ & $\begin{array}{c}20 \\
\mathrm{a}\end{array}$ & $\begin{array}{c}-24.46 \\
\text { a-e }\end{array}$ & $\begin{array}{c}-4.69 \\
\text { a-d }\end{array}$ & $\begin{array}{c}-50.93 \\
a-e\end{array}$ & $\begin{array}{c}25.24 \\
\mathrm{a}\end{array}$ & $\begin{array}{c}26.95 \\
\mathrm{ab}\end{array}$ & $\begin{array}{l}10 \\
\mathrm{ab}\end{array}$ & $\begin{array}{c}-36.67 \\
\text { a-e }\end{array}$ & $\begin{array}{c}69.57 \\
a b\end{array}$ & $\begin{array}{c}-22.5 \\
a-d\end{array}$ & $\begin{array}{c}4.91 \\
\mathrm{ac}\end{array}$ & $\begin{array}{c}-8.29 \\
a b\end{array}$ \\
\hline $1 \times 3$ & $\begin{array}{l}0.8 \\
a b c\end{array}$ & $\begin{array}{c}2.18 \\
n s\end{array}$ & $\begin{array}{c}-18.64 \\
a b\end{array}$ & $\begin{array}{c}-26.83 \\
a b c\end{array}$ & $\begin{array}{c}-48.14 \\
a b c\end{array}$ & $\begin{array}{c}-45.13 \\
a b\end{array}$ & $\begin{array}{c}6.41 \\
\mathrm{ab}\end{array}$ & $\begin{array}{c}14.18 \\
\text { abc }\end{array}$ & $\begin{array}{r}15 \\
\mathrm{a}\end{array}$ & $\begin{array}{c}15.56 \\
\mathrm{a}\end{array}$ & $\begin{array}{c}-17.64 \\
\text { a-e }\end{array}$ & $\begin{array}{c}-15.75 \\
\text { a-e }\end{array}$ & $\begin{array}{c}2.27 \\
a-d\end{array}$ & $\begin{array}{c}-2.27 \\
\mathrm{a}\end{array}$ & $\begin{array}{c}21.88 \\
\text { a-e }\end{array}$ & $\begin{array}{c}-38.75 \\
\text { a-e }\end{array}$ & $\begin{array}{c}16.92 \\
\mathrm{a}\end{array}$ & $\begin{array}{c}6.83 \\
b\end{array}$ \\
\hline $1 \times 4$ & $\begin{array}{c}-2.77 \\
\text { abc }\end{array}$ & $\begin{array}{c}2.46 \\
\mathrm{~ns}\end{array}$ & $\begin{array}{c}42.06 \\
\mathrm{a}\end{array}$ & $\begin{array}{c}33.77 \\
\mathrm{a}\end{array}$ & $\begin{array}{c}-59.68 \\
\text { a-e }\end{array}$ & $\begin{array}{c}-50.68 \\
\text { abc }\end{array}$ & $\begin{array}{c}4 \\
\mathrm{ac}\end{array}$ & $\begin{array}{c}21.14 \\
\mathrm{ab}\end{array}$ & $\begin{array}{l}3.52 \\
\mathrm{abc}\end{array}$ & $\begin{array}{c}-3.84 \\
a b\end{array}$ & $\begin{array}{c}-14.85 \\
a-d\end{array}$ & $\begin{array}{c}20.75 \\
a b c\end{array}$ & $\begin{array}{c}22.73 \\
a\end{array}$ & $\begin{array}{c}-4.81 \\
a b\end{array}$ & $\begin{array}{c}80 \\
\mathrm{a}\end{array}$ & $\begin{array}{c}-10.32 \\
a b\end{array}$ & $\begin{array}{c}-24.63 \\
\text { a-e }\end{array}$ & $\begin{array}{c}-9.75 \\
a b\end{array}$ \\
\hline $2 \times 3$ & $\begin{array}{c}15.33 \\
b\end{array}$ & $\begin{array}{c}11.75 \\
\text { ns }\end{array}$ & $\begin{array}{c}-72.48 \\
a-e\end{array}$ & $\begin{array}{c}-76.34 \\
\text { a-e }\end{array}$ & $\begin{array}{c}-44.35 \\
a b\end{array}$ & $\begin{array}{c}-54.85 \\
a-d\end{array}$ & $\begin{array}{c}2.47 \\
\text { abd }\end{array}$ & $\begin{array}{c}-12.5 \\
\text { a-e }\end{array}$ & $\begin{array}{c}-4.69 \\
\text { abce }\end{array}$ & $\begin{array}{c}-41.67 \\
\text { abce }\end{array}$ & $\begin{array}{c}8.3 \\
a b\end{array}$ & $\begin{array}{c}5.59 \\
\text { a-e }\end{array}$ & $\begin{array}{c}8 \\
a b c\end{array}$ & $\begin{array}{c}-22.5 \\
\text { a-e }\end{array}$ & $\begin{array}{l}-6.25 \\
a-e\end{array}$ & $\begin{array}{c}-34.38 \\
\text { a-e }\end{array}$ & $\begin{array}{c}11.34 \\
b\end{array}$ & $\begin{array}{c}10.05 \\
\mathrm{a}\end{array}$ \\
\hline $2 \times 4$ & $\begin{array}{c}4.43 \\
\mathrm{a}\end{array}$ & $\begin{array}{c}5 \\
\mathrm{~ns}\end{array}$ & $\begin{array}{c}-32.9 \\
\text { a-d }\end{array}$ & $\begin{array}{c}-38.25 \\
\text { abce }\end{array}$ & $\begin{array}{c}-68.67 \\
a-e\end{array}$ & $\begin{array}{c}-62.94 \\
\text { a-e }\end{array}$ & $\begin{array}{c}-1.23 \\
\text { a-d }\end{array}$ & $\begin{array}{c}-7.86 \\
\text { a-d }\end{array}$ & $\begin{array}{c}-12.02 \\
\mathrm{a}-\mathrm{e}\end{array}$ & $\begin{array}{c}-41.3 \\
\text { a-d }\end{array}$ & $\begin{array}{r}3.07 \\
a b c\end{array}$ & $\begin{array}{c}35.47 \\
\mathrm{a}\end{array}$ & $\begin{array}{c}-18.18 \\
\text { a-e }\end{array}$ & $\begin{array}{c}-11.67 \\
a b c\end{array}$ & $\begin{array}{c}52 \\
\mathrm{abc}\end{array}$ & $\begin{array}{c}-14.19 \\
\text { abc }\end{array}$ & $\begin{array}{c}4.56 \\
\mathrm{ad}\end{array}$ & $\begin{array}{c}-5.61 \\
a b c\end{array}$ \\
\hline $3 \times 4$ & $\begin{array}{c}19.61 \\
\mathrm{a}\end{array}$ & $\begin{array}{c}16.45 \\
\text { ns }\end{array}$ & $\begin{array}{c}-25.03 \\
a b c\end{array}$ & $\begin{array}{c}0.03 \\
\mathrm{ab}\end{array}$ & $\begin{array}{c}0.08 \\
\mathrm{a} \\
\end{array}$ & $\begin{array}{c}-70.65 \\
\text { a-e }\end{array}$ & $\begin{array}{l}-3 \\
\text { a-d } \\
\end{array}$ & $\begin{array}{c}22.73 \\
\mathrm{a}\end{array}$ & $\begin{array}{c}12.32 \\
\mathrm{ab}\end{array}$ & $\begin{array}{c}-9.32 \\
a b c\end{array}$ & $\begin{array}{c}-26.35 \\
\text { a-e }\end{array}$ & $\begin{array}{c}11.57 \\
\text { a-d }\end{array}$ & -22.73 a-e & $\begin{array}{c}-13.46 \\
\text { a-d }\end{array}$ & $\begin{array}{c}31.25 \\
\text { a-d }\end{array}$ & $\begin{array}{c}-6.25 \\
\mathrm{a}\end{array}$ & $\begin{array}{c}-1.54 \\
\text { abe }\end{array}$ & $\begin{array}{c}-11.71 \\
\text { abc }\end{array}$ \\
\hline $\begin{array}{l}\text { CD at } \\
5 \%\end{array}$ & 11.70 & 24.76 & 3.13 & 2.94 & 0.05 & 0.04 & 3.49 & 1.52 & 1.28 & 1.00 & 0.39 & 0.19 & 0.90 & 0.35 & 3.01 & 0.98 & 7.29 & 4.75 \\
\hline
\end{tabular}

1: Shammam El-Jordan, 2: ShahdEl-Dokki, 3: Kahera 6 and 4: Charentais. 


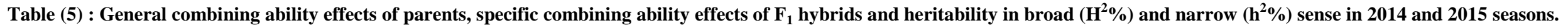

\begin{tabular}{|c|c|c|c|c|c|c|c|c|c|c|c|c|c|c|c|c|c|c|}
\hline \multirow[t]{2}{*}{ Genotype } & \multicolumn{2}{|c|}{ Plant height } & \multicolumn{2}{|c|}{$\begin{array}{c}\text { Total yield per } \\
\text { plant }\end{array}$} & \multicolumn{2}{|c|}{$\begin{array}{l}\text { Average fruit } \\
\text { weight }\end{array}$} & \multicolumn{2}{|c|}{ Fruit length } & \multicolumn{2}{|c|}{ Fruit diameter } & \multicolumn{2}{|c|}{$\begin{array}{l}\text { Fruit shape } \\
\text { index }\end{array}$} & \multicolumn{2}{|c|}{ Flesh thickness } & \multicolumn{2}{|c|}{$\begin{array}{l}\text { Total soluble } \\
\text { solids }\end{array}$} & \multicolumn{2}{|c|}{$\begin{array}{c}\text { Chlorophyll } \\
\text { content in } \\
\text { leaves }\end{array}$} \\
\hline & 2014 & 2015 & 2014 & 2015 & 2014 & 2015 & 2014 & 2015 & 2014 & 2015 & 2014 & 2015 & 2014 & 2015 & 2014 & 2015 & 2014 & 2015 \\
\hline \multicolumn{19}{|c|}{ General combining ability effects } \\
\hline 1 & -26.11 & -28.28 & $\begin{array}{c}-1.4 \\
a b\end{array}$ & -0.76 & -0.05 & $\begin{array}{c}-0.04 \\
a b\end{array}$ & -0.31 & 0.9 & 0.01 & $-0.54 a b c$ & $\begin{array}{c}-0.03 \\
\end{array}$ & $\begin{array}{c}-0.04 \\
n s\end{array}$ & $\begin{array}{c}-0.14 \\
S\end{array}$ & -0.36 & 0.15 & $\begin{array}{c}-0.71 \\
\end{array}$ & -2.82 & $\begin{array}{c}-0.12 \\
n s\end{array}$ \\
\hline 2 & $\begin{array}{l}5.5 \\
\mathrm{ab}\end{array}$ & $\begin{array}{c}5.94 \\
\mathrm{ab}\end{array}$ & $\begin{array}{c}2.38 \\
\mathrm{a}\end{array}$ & $\begin{array}{c}-0.02 \\
a b\end{array}$ & $\begin{array}{c}0.38 \\
\mathrm{a}\end{array}$ & $\begin{array}{c}0.37 \\
\mathrm{a}\end{array}$ & $\begin{array}{c}\mathrm{a} \\
1.97 \\
\mathrm{a}\end{array}$ & $\begin{array}{l}\mathrm{av} \\
1.06 \\
\mathrm{a}\end{array}$ & $\begin{array}{c}-0.29 \\
a\end{array}$ & $\begin{array}{c}1.5 \\
\mathrm{a}\end{array}$ & $\begin{array}{c}0.22 \\
\mathrm{a}\end{array}$ & $\begin{array}{c}-0.04 \\
n s\end{array}$ & $\begin{array}{c}0.1 \\
\mathrm{~ns}\end{array}$ & 0.27 & $\begin{array}{l}-1.13 \\
a b\end{array}$ & -0.81 & 0.39 & -0.58 \\
\hline 3 & $\begin{array}{c}19.17 \\
\mathrm{a}\end{array}$ & $\begin{array}{c}18.89 \\
\mathrm{a}\end{array}$ & $\begin{array}{c}-2.74 \\
a b\end{array}$ & $\begin{array}{c}-1.48 \\
a b\end{array}$ & $\begin{array}{c}-0.05 \\
\mathrm{a}\end{array}$ & $\begin{array}{c}-0.05 \\
\text { ac }\end{array}$ & $\begin{array}{c}-0.89 \\
a\end{array}$ & $\begin{array}{l}-0.77 \\
a b\end{array}$ & $\begin{array}{c}-0.36 \\
a\end{array}$ & $-1.08 a b c$ & $\begin{array}{c}-0.03 \\
a\end{array}$ & $\begin{array}{c}0.05 \\
\mathrm{~ns}\end{array}$ & $\begin{array}{c}-0.18 \\
n s\end{array}$ & $\begin{array}{l}-0.12 \\
-0.12\end{array}$ & 0.49 & $\begin{array}{l}0.43 \\
a b\end{array}$ & $\begin{array}{c}-0.23 \\
n s\end{array}$ & $\begin{array}{c}-0.64 \\
n s\end{array}$ \\
\hline 4 & $\begin{array}{c}1.44 \\
\mathrm{ac}\end{array}$ & $\begin{array}{c}3.44 \\
\mathrm{ac}\end{array}$ & $\begin{array}{c}1.76 \\
\mathrm{~b}\end{array}$ & $2.27 \mathrm{a}$ & $\begin{array}{c}-0.29 \\
a\end{array}$ & $\begin{array}{l}-0.28 \\
a b c\end{array}$ & $\begin{array}{c}-0.78 \\
a\end{array}$ & $\begin{array}{c}0.61 \\
\mathrm{~b}\end{array}$ & $\begin{array}{c}0.64 \\
\mathrm{a}\end{array}$ & $0.12 \mathrm{ab}$ & $\begin{array}{c}-0.16 \\
a\end{array}$ & $\begin{array}{c}0.02 \\
\text { ns }\end{array}$ & $\begin{array}{c}0.22 \\
\text { ns }\end{array}$ & $\begin{array}{c}0.21 \\
\mathrm{~b}\end{array}$ & $\begin{array}{c}0.49 \\
\mathrm{~b}\end{array}$ & $\begin{array}{c}1.09 \\
\mathrm{a}\end{array}$ & $\begin{array}{c}2.66 \\
\mathrm{a}\end{array}$ & $\begin{array}{c}1.34 \\
\mathrm{~ns}\end{array}$ \\
\hline $\begin{array}{c}\text { CD at } 5 \% \\
\left(g_{i}-g_{j}\right)\end{array}$ & 5.52 & 11.67 & 1.48 & 1.39 & 0.02 & 0.02 & 1.65 & 0.72 & 0.6 & 0.47 & 0.18 & 0.09 & 0.42 & 0.17 & 1.42 & 0.46 & 3.44 & 2.24 \\
\hline \multicolumn{19}{|c|}{ Specific combining ability effects } \\
\hline $1 \times 2$ & $\begin{array}{c}46.88 \\
b\end{array}$ & $\begin{array}{c}53.07 \\
\mathrm{a}\end{array}$ & $\begin{array}{c}-0.53 \\
\mathrm{a}\end{array}$ & $\begin{array}{c}0.03 \\
\mathrm{ac}\end{array}$ & $\begin{array}{c}-0.36 \\
a-e\end{array}$ & $\begin{array}{l}-0.35 \\
a-e\end{array}$ & $\begin{array}{c}3.33 \\
\mathrm{a}\end{array}$ & $\begin{array}{c}-1.78 \\
\mathrm{a}-\mathrm{e}\end{array}$ & $\begin{array}{c}-0.23 \\
a b\end{array}$ & $-3.51 \mathrm{a}$ & $\begin{array}{c}0.35 \\
\mathrm{a}\end{array}$ & $0.25 \mathrm{a}$ & $\begin{array}{c}0.44 \\
\mathrm{~b}\end{array}$ & $\begin{array}{c}-0.48 \\
a b\end{array}$ & $2.89 \mathrm{~b}$ & $-0.73 \mathrm{a}$ & $\begin{array}{r}1.5 \\
\mathrm{~d}\end{array}$ & $\begin{array}{c}-2.81 \\
a b\end{array}$ \\
\hline $1 \times 3$ & $\begin{array}{l}6.21 \\
a b c\end{array}$ & $\begin{array}{c}17.12 \\
\mathrm{ad}\end{array}$ & $\begin{array}{c}-0.49 \\
a\end{array}$ & $\begin{array}{l}-1.01 \\
\mathrm{ab}\end{array}$ & $\begin{array}{c}-0.28 \\
\text { a-d }\end{array}$ & $\begin{array}{c}-0.28 \\
a-d\end{array}$ & $\begin{array}{c}0.03 \\
\mathrm{~ns}\end{array}$ & $\begin{array}{l}0.97 \\
\text { ac }\end{array}$ & $1.18 \mathrm{~b}$ & $2.37 \mathrm{ab}$ & -0.16 & $\begin{array}{l}-0.19 \\
a-e\end{array}$ & $0.05 \mathrm{a}$ & $\begin{array}{c}0.24 \\
\mathrm{~b}\end{array}$ & $\begin{array}{c}1.28 \\
\mathrm{~ns}\end{array}$ & $\begin{array}{c}-1.64 \\
a b c\end{array}$ & $\begin{array}{c}4.79 \\
\mathrm{a}\end{array}$ & $\begin{array}{c}3.82 \\
\mathrm{a}\end{array}$ \\
\hline $1 \times 4$ & $\begin{array}{c}-2.07 \\
a b c\end{array}$ & $\begin{array}{c}16.57 \\
\text { abe }\end{array}$ & $4.93 \mathrm{a}$ & $4.63 \mathrm{a}$ & $\begin{array}{c}-0.21 \\
a b c\end{array}$ & $\begin{array}{c}-0.21 \\
a b c\end{array}$ & $\begin{array}{c}-0.58 \\
\mathrm{~ns}\end{array}$ & $\begin{array}{c}2.33 \\
b\end{array}$ & $\begin{array}{c}0.44 \\
\mathrm{ac}\end{array}$ & $0.74 \mathrm{ac}$ & $\begin{array}{c}-0.11 \\
\mathrm{a}\end{array}$ & $\begin{array}{l}0.12 \\
\text { abe }\end{array}$ & $1.15 \mathrm{a}$ & $\begin{array}{c}0.35 \\
\mathrm{a}\end{array}$ & $3.28 \mathrm{a}$ & $0.44 \mathrm{a}$ & $\begin{array}{r}-6.3 \\
a-e\end{array}$ & $\begin{array}{l}-1.67 \\
a b\end{array}$ \\
\hline $2 \times 3$ & $\begin{array}{c}28.93 \\
\text { abc }\end{array}$ & $\begin{array}{c}19.57 \\
\text { ac }\end{array}$ & $\begin{array}{c}-2.75 \\
a b\end{array}$ & $\begin{array}{c}-3.25 \\
a b c\end{array}$ & $\begin{array}{c}-0.14 \\
a b\end{array}$ & $\begin{array}{c}-0.13 \\
a b\end{array}$ & $\begin{array}{c}1.25 \\
\mathrm{a}\end{array}$ & $\begin{array}{c}0.32 \\
\text { abd }\end{array}$ & $0.14 \mathrm{a}$ & $-1.31 \mathrm{a}-\mathrm{d}$ & $\begin{array}{c}0.09 \\
\mathrm{~ns}\end{array}$ & $\begin{array}{c}0.12 \\
\text { abd }\end{array}$ & $0.41 \mathrm{c}$ & $\begin{array}{c}-0.16 \\
a b\end{array}$ & $\begin{array}{c}-0.44 \\
a b\end{array}$ & $\begin{array}{c}-1.08 \\
a b c\end{array}$ & $\begin{array}{c}1.45 \\
\mathrm{e}\end{array}$ & $\begin{array}{c}3.51 \\
b\end{array}$ \\
\hline $2 \times 4$ & $\begin{array}{c}-7.68 \\
a b c\end{array}$ & $\begin{array}{c}-8.32 \\
a-e\end{array}$ & $\begin{array}{c}-1.62 \\
a\end{array}$ & $\begin{array}{c}-3.08 \\
a b c\end{array}$ & $\begin{array}{c}-0.48 \\
a-e\end{array}$ & $\begin{array}{c}-0.47 \\
a-e\end{array}$ & $\begin{array}{c}-0.03 \\
a\end{array}$ & $\begin{array}{l}-0.19 \\
\text { abe }\end{array}$ & $\begin{array}{c}-1.04 \\
a b c\end{array}$ & -2.43 a-e & $\begin{array}{c}0.13 \\
\mathrm{~ns}\end{array}$ & $0.23 \mathrm{~b}$ & $\begin{array}{c}-0.59 \\
a b c\end{array}$ & $\begin{array}{c}-0.05 \\
\mathrm{a}\end{array}$ & $\begin{array}{c}2.22 \\
\mathrm{~ns}\end{array}$ & $0.14 \mathrm{~b}$ & $\begin{array}{c}4.36 \\
\mathrm{~b}\end{array}$ & $\begin{array}{c}0.75 \\
\mathrm{~ns}\end{array}$ \\
\hline $3 \times 4$ & $\begin{array}{c}48.99 \\
\mathrm{a}\end{array}$ & $\begin{array}{c}40.07 \\
\mathrm{~b}\end{array}$ & $\begin{array}{c}0.45 \\
\mathrm{ab}\end{array}$ & $1.98 \mathrm{~b}$ & $0.31 \mathrm{a}$ & $0.31 \mathrm{a}$ & $\begin{array}{c}0.5 \\
\mathrm{a}\end{array}$ & $2.43 \mathrm{a}$ & $1.8 \mathrm{a}$ & $\begin{array}{c}0.61 \\
\text { a-e }\end{array}$ & $\begin{array}{c}-0.16 \\
\mathrm{a}\end{array}$ & $\begin{array}{c}0.15 \\
\mathrm{ac}\end{array}$ & $\begin{array}{c}-0.48 \\
a b c\end{array}$ & $\begin{array}{c}-0.2 \\
a b\end{array}$ & $\begin{array}{c}1.94 \\
\mathrm{~ns}\end{array}$ & $0.02 \mathrm{c}$ & $\begin{array}{c}2.08 \\
\mathrm{c}\end{array}$ & $\begin{array}{l}-2.08 \\
a b\end{array}$ \\
\hline $\begin{array}{c}\text { CD at 5\% } \\
\left(s_{i j}-s_{k l}\right)\end{array}$ & 11.03 & 23.34 & 2.95 & 2.77 & 0.04 & 0.04 & 3.29 & 1.43 & 1.2 & 0.94 & 0.37 & 0.18 & 0.84 & 0.33 & 2.83 & 0.93 & 6.87 & 4.48 \\
\hline \multicolumn{19}{|c|}{ Heritability in broad and narrow sense } \\
\hline $\mathbf{H}^{2} \%$ & 0.15 & 0.09 & 0.03 & 0.05 & 0.06 & 0.06 & 0.01 & 0.07 & 0.06 & 0.26 & 0.02 & 0.12 & 0.04 & 0.03 & 0.46 & 0.03 & 0.02 & 0.01 \\
\hline$h^{2} \%$ & 0.02 & 0.00 & 0.02 & 0.01 & 0.02 & 0.02 & 0.01 & 0.01 & 0.01 & 0.01 & 0.01 & 0.05 & 0.01 & 0.02 & 0.16 & 0.02 & 0.00 & 0.00 \\
\hline
\end{tabular}

1: Shammam El-Jordan, 2: Shahd El-Dokki, 3: Kahera 6 and 4: Charentais. 
$\times$ Kahera 6 for fruit diameter, Shammam ElJordan $\times$ Charantais for total yield per plant and Kahera $6 \times$ Charentais for plant height. The range of increase in the hybrids over high-parents was between 15\% (Shammam El-Jordan $\times$ Kahera 6 for fruit diameter) to $42.06 \%$ (Shammam ElJordan $\times$ Charantais for total yield per plant) in both 2014 and 2015 seasons. These results are in agreement with those obtained by Kitroongruang et al. (1992) and Ramaswamy et al. (1977) they recorded high heterosis estimates for melon for fruit diameter, fruit length, fruit shape index, the number of fruits per plant, total fruit weight per plant, fruit weight and percentage total soluble solids content.

The GCA and SCA effects and heritability estimates in narrow and broad sense are presented in Table (5). Out of the 4 parents, the cultivar Shahd ElDokki had the highest GCA effect in both seasons for average fruit weight and fruit length and cv Charentais had the highest values for total soluble solids and chlorophyll content. High positive SCA effects were found in the crosses Shammam El-Jordan $\times$ Shahd El-Dokki for fruit shape index, Shammam El-Jordan $\times$ Kahera 6 for chlorophyll content, Shammam El-Jordan $\times$ Charentais for total yield per plant, fruit thickness and total soluble solids and Kahera $6 \times$ Charentais for average fruit weight. These results indicated that no cross combinationis consistently good for all characters. These results agreed with the results of Kitroongruang et al. (1992) and Ramaswamy et al. (1977) who reported high GCA and positive SCA for yield, fruit weight, fruit diameter and fruit length in melon.

Heritability values in broad and narrow sense are shown in Table (5). In respect to broad sense heritability, the values were low for all the studied characters in both 2014 and 2015 seasons. Also, the values of narrow sense heritability were low for all the studied characters in both seasons indicating that non-additive gene effects had the major role in inheritance of these characters. The results are in agreement with the results of Reddy et al. (2013) and Rakhi and Rajamony (2005) on melon who found low values of heritability for some yield components.

The SCA effects of the crosses Shammam ElJordan $\times$ Charentais and Kahera $6 \times$ Charentais were correlated with their GCA parent effects. The two crosses were the best cross combination in three characters, viz., total yield per plant, flesh thickness and total soluble solids in the cross Shammam El-Jordan $\times$ Charentais and in twocharacters, viz., plant height and average fruit weight in the cross Kahera $6 \times$ Charentais. In addition, the two crosses involved at least one parent with high GCA effects. Thus, these two crosses have the availability to be exploited in future breeding program to produce new local hybrids of melon.

It could be concluded that high GCA can be used to choose the parents with desirable characters to produce new local hybrids. The parents with desirable characters and high GCA may produce hybrids with high heterosis and SCA. According to that in the current study the best crosses which can be produced as new local hybrids of melon are Shammam El-Jordan $\times$ Charentais and Kahera $6 \times$ Charentais.

\section{REFERENCES}

Duvick D. N. (1999). Heterosis: Feeding people and protecting natural resources. In: Coors J.G. and Pandey S. Editors. The genetics and exploitation of heterosis in crops. Amer. Soc. Agron. Inc., Madison, WI, USA, 19$30 \mathrm{p}$.

Fernandez-Silva I., Moreno E., Eduardo I., Arus P., Alvarez J.M. and Monforte A.J. (2009). On the genetic control of heterosis for fruit shape in melon (Cucumis melo L.). J. Heredity, 100 (2): 229 -235.

Feyzian E., Dehghani H., Rezai A. M. and Jalali J. M. (2009a). Cross analysis for maturity and yield-related traits in melon (Cucumis melo L.). Euphytica, 168(2): 215 - 223.

Feyzian F., Dehghani H., Rezai A. M. and Jalali J. M. (2009b). Correlation and sequential path model for some yield-related traits in melon (Cucumis meloL.). J. Agric. Sci. Technol. 11: 341-353.

Greish S. M., El-Foly A.H. M., Abd El-Raheem A. A. and Guirgis A.A. (2005). General and specific combining ability and expression of heterosis in some Cucumis melo crosses. Zagazig J. Agric., 32(5): 1501 - 1515.

Griffing B. (1956). Concepts of general and specific combining ability in relation to diallel crossing systems. Aust. J. Biol. Sci., 
9: 463 - 493.

Hatem A. K. (1992). Genetic and physiological behaviour of some characters in melon (Cucmis melo L.). Ph. D. Thesis Fac. of Agric. Menufiya University, Egypt, 119 p.

Hatem A. K., Shaheen H. A. and El-Doweny H. H. (1995). Combining ability for some economic useful characters in melon. Menufiya J. Agric. Res., 20(6): 2331 2348.

Kitroongruang N., Poo-Swang W. and Tokumasu S. (1992). Evaluation of combining ability, heterosis and genetic variance for plant growth and fruit quality characteristics in Thai-melon (Cucurnis melo L., var. acidulous Naud.). Sci. Hort., 50: 79-87.

Kupper R. S. and Jack E. S.(1988) Combining ability and (Cucumis sativus var hardwickii ( R )Alef.

Luan F., Sheng Y., Wang Y. and Staub J.E. (2010). Performance of melon hybrids derived from parents of diverse geographic origins. Euphytica, 173: 1-16.
Rakhi R. and Rajamony L. (2005). Variability, heritability and genetic advance in landraces of culinary melon (Cucumis melo L.). J. Tropical Agric., 43 (1-2): 79-82.

Ramaswamy B., Seshadri V. S. and Sharma J. C. (1977). Inheritance of some fruit characters in muskmelon. Sci. Hort., 6: 107-120.

Reddy B. P. K., Begum H., Sunil N. and Reddy M. T. (2013). Variance component analysis of quantitative traits in muskmelon (Cucumis melo L.). Trakia J. Sci., 11(2): 118-124.

Singh R. K. and Chaudhary B. D. (1977). Biometrical methods in quantitative genetic analysis. Kalyani Publishers, Ludhiana, India, 304 pp.

Snedecor G.W. and Cochran W.G. (1980). Statistical Methods, $7^{\text {th }}$ ed. Iowa State Univ. Press, Iowa, USA, $491 \mathrm{pp}$.

Valérioet I. P., Carvalho F. I. F., Oliveira A. C., Souza Ribeiro G., Nornberg R. and Lach H. (2009). Genotypes in two models of diallel analysis. Biotechnology. 9: 100-107.

\footnotetext{
سلوك أربعة آباء من الثمام وهجنهم الستة

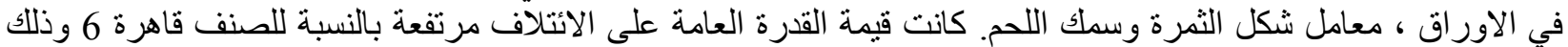

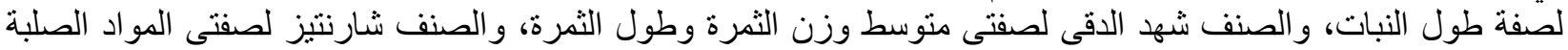

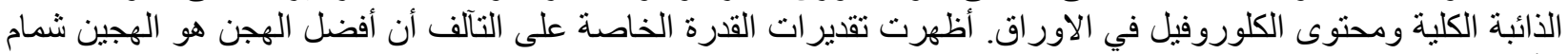

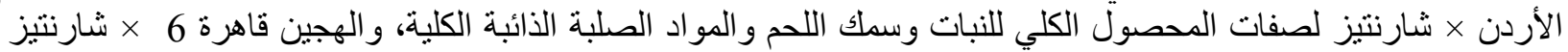
لصفتى طول النبات ومتوسط وزن الثنرة الثرة قيم الكفاءة التوريثية في كل من معناها الواسع و الضيق كانت منخفضة لكل الصفات المدروسة في كلا موسمى الدر استة

المجلة العلمية لكلية الزراعة -جامعة القاهرة ـ المجلا (67) العدد الثالث (يوليو 2016 ) :264-257 ـ الثيل
} 